\title{
Validación de un cuestionario de mejora de la satisfacción laboral (CMSL) en profesionales de atención primaria
}

\section{Validation of a questionnaire on improving work satisfaction (CMSL) in primary care professionals}

\author{
I. Pérez-Ciordiaa ${ }^{1,2}$, F. Guillén-Grima ${ }^{2,3}$, A. Brugos Larumbe ${ }^{1,2}$, I. Aguinaga Ontoso², \\ C. Fernández-Martínez ${ }^{1}$
}

\section{RESUMEN}

Fundamento. La satisfacción laboral de los profesionales sanitarios se considera un indicador de calidad en la gestión del sistema, estando relacionado con la eficacia de los servicios ofrecidos.

El objetivo del estudio es la validación de un cuestionario para evaluar la mejora de la satisfacción laboral (CMSL) en una población de profesionales sanitarios de atención primaria en Navarra.

Metodología. Se realizó un estudio descriptivo con cuestionarios autocumplimentados, siendo la población diana todos los profesionales de atención primaria (médicos, pediatras y personal de enfemería) de los centros de salud de Navarra. Se utilizó la escala Lickert para la medición de los ítems. Se recogieron datos descriptivos de sexo, años de ejercicio profesional, satisfacción laboral, estamento profesional, centro de salud y 47 ítems sobre mejora de satisfacción laboral. Se calculó la fiabilidad mediante el coeficiente alfa de Cronbach y se halló la validez de constructo mediante un análisis factorial con rotación varimax, agrupando los ítems en 9 dimensiones.

Resultados. Se recogieron un total de 414 cuestionarios. Se obtuvo el coeficiente alfa de Cronbach, con un valor global de 0,933 . Entre 5 dimensiones se explica el $41,287 \%$ de la varianza total. La dimensión "relaciones con los pacientes" presentó la media $(4,087)$ más alta de mejora de la satisfacción laboral y el ítem "Si pudiera eliminar la demanda injustificada" presentó una media de 4,21.

Conclusiones. El cuestionario diseñado es un instrumento válido para la evaluación integral de la mejora de la satisfacción laboral de los profesionales de atención primaria. Los resultados obtenidos pueden orientar sobre qué áreas de mejora se deben implantar para mejorar la satisfacción de los profesionales.

Palabras clave. Satisfacción laboral. Estudios de validación. Atención primaria. \begin{abstract}
Background. Job satisfaction of health professionals is considered to be a quality indicator, as it is related to the efficacy of the services.

The aim of the study is to validate a questionnaire for evaluating job satisfaction improvement in a population of health professionals in primary care in Navarre.
\end{abstract}

Methodology. Descriptive study with self-completed questionnaires; the target population was all health care professionals (family doctors, pediatricians and nurses) of primary health centers of Navarre. A Lickert scale was used for measuring the items. Other variables measured were: sex, years in the profession, job satisfaction, professional status, health center, and 47 items on improving job satisfaction. Cronbach's alpha coefficient was used to evaluate reliability, and to evaluate construct validity factor analysis with varimax rotation, grouping the items in 9 dimensions was used.

Results. A total of 414 questionnaires were collected Cronbach's alpha coefficient was 0.933 . Forty-one point two eight seven percent (41.287\%) of total variance was explained by five dimensions. The dimension "relations with patients" presented the highest average (4.087) of improvement in job satisfaction, and the item "If it were possible to eliminate unjustified demand" showed an average of 4.21 .

Conclusions. The questionnaire designed is a valid instrument for a comprehensive evaluation of the improvement in the job satisfaction of primary care professionals. The results obtained can indicate which areas of improvement should be implemented in order to improve the satisfaction of the professionals.

Key words. Job satisfaction. Validation studies. Primary care.
1. Servicio Navarro de Salud-Osasunbidea. Pamplona. Navarra.

2. Departamento de Ciencias de la Salud. Universidad Pública de Navarra. Pamplona. Navarra.

3. Unidad Medicina Preventiva. Clínica Universidad de Navarra. Pamplona. Navarra.

\section{Correspondencia:}

Francisco Guillén Grima

Departamento de Ciencias de la Salud

Universidad Pública de Navarra

Avda. de Barañain

31008-Pamplona

Recepción: 14 de marzo de 2012

Aceptación provisional: 26 de abril de 2012

Aceptación definitiva: 28 de mayo de 2012. 


\section{INTRODUCCIÓN}

La eficacia de los servicios ofrecidos por un sistema sanitario está relacionado con el nivel de satisfacción de los profesionales que en él trabajan ${ }^{1-3}$.

Se afirma que es imposible satisfacer las expectativas del cliente externo, del usuario, sin satisfacer las del profesional o cliente interno ${ }^{4}$.

No existe una definición unánimemente aceptada de satisfacción laboral, siendo definida como una respuesta afectiva del trabajador hacia diferentes aspectos de su trabajo, estando condicionada tanto por las circunstancias del trabajo como por las características de cada persona ${ }^{5}$.

La satisfacción laboral se ha vinculado con variables como la profesión, reconocimiento laboral, responsabilidad, condiciones propias del trabajo, formación continuada, tiempo adecuado con los pacientes, equidad en los premios, relaciones interpersonales y otros ${ }^{6-7}$. Por el contrario, la insatisfacción laboral se da cuando concurren circunstancias como trabajo excesivo, estrés laboral, pérdida de autonomía clínica, bajo salario y todos aquellos otros factores inversos respecto al punto de satisfacción laboral ${ }^{8-11}$.

Determinar si un trabajador está o no desgastado profesionalmente no es fácil, siendo un proceso continuado en el tiempo. La satisfacción laboral y su opuesto o desgaste profesional constituyen problemas de origen multicausal.

Mediante los indicadores de satisfacción se pretende diagnosticar a una población laboral para ver si ésta presenta algún mal y éste es remediable, o bien si todo funciona de manera correcta.

La insatisfacción laboral conlleva la desmoralización y disminución de la calidad en la atención sanitaria, afectando tanto al profesional como al paciente y a la institución de salud, manteniendo una relación directa con el absentismo ${ }^{12}$ y un déficit en la relación profesional médico-paciente.

Mantener la insatisfacción laboral, sin atender las necesidades del profesional, puede dar como resultado el síndrome de desgaste profesional entendido como un estado de frustración que se produce por la dedicación a una causa que no produce el refuerzo esperado o como una respuesta inadecuada al estrés laboral crónico, además de por el contacto con el sufrimiento ${ }^{13}$.

Existen factores organizacionales y funcionales que pueden ser los que generen insatisfacción en el personal ${ }^{14-15}$, existiendo actualmente actuaciones de política sanitaria que reconocen la importancia de mejorar la satisfacción de los profesionales de la salud ${ }^{16-17}$.

Las escalas, al igual que todos los instrumentos de medición, deben ser válidos y fiables ${ }^{18}$. La validez se refiere a la capacidad del instrumento para medir el constructo ${ }^{19}$ y la confiabilidad a la propiedad de mostrar resultados similares en mediciones repetidas y a la propiedad de homogeneidad o consistencia ${ }^{20-22}$.

La investigación se ha desarrollado con el objeto de aportar un instrumento para la evaluación integral de la mejora de la satisfacción laboral en profesionales de atención primaria.

El objetivo del presente trabajo es el desarrollo y validación de un cuestionario para evaluar la mejora de la satisfacción laboral (CMSL) en una población de profesionales sanitarios de atención primaria en Navarra.

\section{SUJETOS Y MÉTODO}

El equipo investigador contactó con una persona del equipo de atención primaria (EAP), mayoritariamente el director del equipo, a quien se le remitieron los cuestionarios por correo junto con una carta de presentación explicando el objetivo del estudio y solicitando la colaboración de los profesionales médicos, de enfermería y pediatras, quienes constituyen la población de estudio. Previamente se había contactado telefónicamente solicitando su disponibilidad y cooperación.

Los cuestionarios fueron remitidos por correo a todos los centros de salud de atención primaria de Navarra con fecha 15 de junio de 2010, facilitándose así mismo dos direcciones de correo para su remisión, una vez cumplimentados. 
El responsable del EAP presentó el estudio de investigación a los compañeros, distribuyó los cuestionarios y realizó su recogida tras un periodo de 15 días dado para su cumplimentación, la cual se realizó de manera anónima.

Transcurrido el plazo dado, se solicitaron los cuestionarios, si previamente no se habían recibido, a los directores del EAP, ampliando 2 semanas más el plazo si fuera necesario. Se solicitó asimismo a cada EAP que remitieran el número de cuestionarios entregados y el número de cuestionarios recogidos.

El proceso metodológico seguido en la elaboración del cuestionario se realizó en 6 fases. En la fase 1 se concretó el aspecto a estudiar (mejora de la satisfacción laboral), la técnica de medida concreta (escala tipo Lickert) y un banco inicial de ítems. Estos ítems se obtuvieron a partir de los cuestionarios Job Description Index (JDI), Minnesota Satisfaction Questionnaire (MSQ) ${ }^{23}$, el cuestionario de Font-Roja ${ }^{24}$, el cuestionario de satisfacción laboral de profesionales de la salud en Equipos de Atención Primaria $\left(\right.$ CSLPS-EAP/33) ${ }^{25}$, el cuestionario de Calidad de vida profesional (CVP-35) ${ }^{26}$, del Modelo Europeo $\mathrm{EFQM}^{27}$, del cuestionario Maslach burnout inventory ${ }^{28} \mathrm{y}$ otros ${ }^{29-31}$, además de aquellos de elaboración propia.

En la fase 2 se realizó la validez de contenido, mediante una depuración de los ítems iniciales, realizada por 5 profesionales expertos en el ámbito de la atención primaria y de gestión. Se eliminaron aquellos ítems que presumiblemente no servirían para evaluar la mejora de la satisfacción, e igualmente se mejoró la redacción de los ítems.

En la fase 3 se asignaron valores a los ítems, optando por 5 categorías: totalmente de acuerdo (5), de acuerdo (4), indiferente (3), en desacuerdo (2), totalmente en desacuerdo (1). Si un ítem no era respondido, se daba 0 puntos.

Para cada ítem se comprobó su capacidad discriminante, comparando si el valor obtenido por el $25 \%$ de los sujetos con mayor puntuación total (percentil 75) en las escalas era significativamente diferente del obtenido por el $25 \%$ de la muestra con me- nor puntuación total (percentil 25). Para el contraste estadístico del poder discriminatorio de cada pregunta se utilizó la prueba no paramétrica U de Mann-Whitney.

Se analizó la correlación de cada ítem con el total de la escala menos ese ítem concreto, mediante la correlación de Pearson, eliminándose aquellos ítems con un coeficiente de correlación inferior a 0,25 con la escala global.

En la fase 4 se calculó la fiabilidad mediante la consistencia interna del cuestionario (valores de 0 a 1 ). Se utilizó el método de las dos mitades y el coeficiente alfa de Cronbach (21) que se emplea para evaluar la homogeneidad de los distintos ítems de una misma dimensión y del cuestionario global (varianza ítem-ítem total).

En la fase 5 se halló la validez de constructo, para lo que se determinó la tendencia de las puntuaciones a agruparse en factores consistentes mediante un análisis factorial con rotación varimax. Se seleccionaron factores con autovalores superiores a 1 que aportaran una explicación de la varianza como mínimo del 3,5\%; la asignación de los ítems a cada factor se realizó considerando que tuviera una saturación con el factor de como mínimo $0,40^{32} \mathrm{y}$, asignando cada ítem a un único factor, aquel con el que tuviera mayor saturación. La adecuación del análisis factorial se comprobó mediante la medida de Kaiser-Meyer-Olkin ${ }^{33}$ y la prueba de esfericidad de Bartlett ${ }^{34}$ para valorar la adecuación de la matriz de datos creada.

En la fase 6 se analizaron las escalas por profesión, sexo, tiempo de actividad asistencial y puntuación de satisfacción laboral. Se utilizó la correlación de Sperman para determinar la relación entre las variables.

\section{RESULTADOS}

En la fase 1 se elaboraron 53 ítems a partir de la revisión bibliográfica y de experiencia individual, eliminándose (fase 2) un total de 6 ítems por 5 jueces-expertos. Sus sugerencias permitieron la corrección y mejora en la redacción de los ítems, quedando confeccionado el cuestionario definitivo con un total de 47 ítems (Anexo 1). 
Se recogieron un total de 432 cuestionarios, con una tasa de respuesta de $62,3 \%$ sobre el total de cuestionarios entregados. Se descartaron 18 cuestionarios por estar las escalas incompletas, siendo incluidos en el análisis 414 cuestionarios. La satisfacción laboral obtuvo un valor medio de 6,7 puntos (escala $1-10$ ) y el $42 \%$ de los en- cuestados tenía una antigüedad en el trabajo superior a 25 años.

Se calculó la media, la desviación típica (DT) y la correlación corregida de cada ítem (Tabla 1). Ningún ítem fue eliminado por su bajo poder discriminante o por su baja correlación con el total del cuestionario, puntuando en todos los casos por encima de 0,300.

Tabla 1. Media, desviación típica (DT) y coeficiente de correlación discriminante (r) de los ítems

\begin{tabular}{|c|c|c|}
\hline Dimensiones e ítems & Media (DT) & $\mathbf{r}\left({ }^{*}\right)$ \\
\hline Competencia profesional (Dimensión 1) & $3,950(0,762)$ & \\
\hline 7. Si dedicara un tiempo mínimo, en horario laboral, para mi formación & $3,90(0,36)$ & 0,414 \\
\hline 8. Si pudiera actuar en actividades de investigación & $3,78(0,86)$ & 0,339 \\
\hline 12. Si mis relaciones con atención especializada fueran mas ágiles y fluidas & $4,15(0,72$ & 0,526 \\
\hline 21. Si tuviera facilidad de mis jefes para acudir a congresos-actividades de formación & $4,02(0,77)$ & 0,448 \\
\hline 23. Si pudiera trabajar más con programas y menos con demanda de atención aguda & $3,32(0,88)$ & 0,459 \\
\hline 25. Si pudiera disponer de más tiempo para cada paciente & $4,11(0,79)$ & 0,498 \\
\hline $\begin{array}{l}\text { 26. Si las cargas de trabajo entre los miembros del equipo (médico-enfermera) fue- } \\
\text { ran más equitativas }\end{array}$ & $3,90(0,88)$ & 0,422 \\
\hline 27. Si trabajáramos en equipo & $4,00(0,83)$ & 0,473 \\
\hline 34. Si tuviera premios y/o reconocimiento a la labor bien desarrollada & $3,80(0,84)$ & 0,398 \\
\hline 35. Si hubiera coordinación entre servicios sanitarios y servicios sociales & $4,20(0,74)$ & 0,474 \\
\hline 36. Si pudiera trabajar sin estrés, sin prisas ni agobios & $4,04(0,69)$ & 0,599 \\
\hline 38. Si pudiera ser mas ágil en aportar solución a mis pacientes & $3,94(0,77)$ & 0,582 \\
\hline 41. Si mis propuestas fueran escuchadas y estudiadas-aplicadas por mis superiores & $3,93(0,78)$ & 0,560 \\
\hline 43. Si hubiera planes de formación en farmacoterapia & $3,94(0,72)$ & 0,477 \\
\hline 44. Si hubiera modelos de seguimiento de los pacientes polimedicados & $3,96(0,69)$ & 0,405 \\
\hline $\begin{array}{l}\text { 47. Si pudiera contactar con especialistas que puedan resolver dudas "en el mo- } \\
\text { mento" }\end{array}$ & $4,18(0,72)$ & 0,570 \\
\hline
\end{tabular}

\section{Satisfacción intrínseca por la actividad laboral (Dimensión 2)}

$3,721(0,883)$

\begin{tabular}{lrr}
\hline $\begin{array}{l}\text { 9. Si pudiera eliminar "de mi cupo" unos pocos pacientes con quienes no logro } \\
\text { empatizar }\end{array}$ & $3,12(1,08)$ & 0,382 \\
\hline 13. Si mis pacientes valoraran el trabajo y el esfuerzo & $3,98(0,76)$ & 0,629 \\
\hline 16. Si fuera capaz de trabajar sin hacer una medicina defensiva & $3,88(0,83)$ & 0,607 \\
\hline 22. Si sintiera un menor control (gasto farmacia) de mis superiores & $3,45(0,98)$ & 0,411 \\
\hline 24. Si los pacientes no "me presionaran" para realizar actividades que considero & $3,84(0,87)$ & 0,570 \\
\hline injustificadas & $3,47(0,90)$ & 0,482 \\
\hline 28. Si mi trabajo no fuera tan monótono (mismos pacientes y mismos problemas) & $3,59(0,85)$ & 0,526 \\
\hline 29. Si tuviera flexibilidad para las agendas de citación & $3,95(0,92)$ & 0,523 \\
\hline 30. Si pudiera eliminar los hiperfrecuentadores & $3,74(0,89)$ & 0,615 \\
\hline 31. Si pudiera trabajar en un ambiente tranquilo, sin amenazas ni coacciones & & \\
\hline
\end{tabular}




\begin{tabular}{|c|c|c|}
\hline Dimensiones e ítems & Media (DT) & $\mathbf{r}\left({ }^{*}\right)$ \\
\hline 32. Si pudiera desconectar al acabar la jornada & $3,53(0,89)$ & 0,543 \\
\hline 33. Si mis pacientes fueran, realmente, con problemas de salud & $3,62(0,86)$ & 0,561 \\
\hline 37. Si pudiera conciliar vida laboral y familiar & $3,99(0,85)$ & 0,520 \\
\hline 45. Si pudiera eliminar la demanda injustificada & $4,21(0,77)$ & 0,567 \\
\hline Relaciones interpersonales con los compañeros (Dimensión 3) & $3,591(0,897)$ & \\
\hline 3. Si mi relación con los compañeros médicos/enfermeras del EAP fuera mejor & $3,616(0,910)$ & 0,345 \\
\hline 4. Si mi relación con otros compañeros no médicos/enfermeros del EAP fuera mejor & $3,567(0,885)$ & 0,365 \\
\hline Satisfacción con la gestión del trabajo (Dimensión 4) & $3,965(0,807)$ & \\
\hline 39. Si pudiera agilizar las listas de espera para pruebas diagnósticas & $4,11(0,72)$ & 0,468 \\
\hline 40. Si pudiera agilizar las consultas con especialistas & $4,12(0,76)$ & 0,499 \\
\hline Retribución y promoción profesional (Dimensión 5) & $3,624(0,931)$ & \\
\hline 1. Si mi sueldo fuera superior & $3,845(1,039)$ & 0,377 \\
\hline 2. Si hubiera posibilidades de promoción & $3,857(0,833)$ & 0,414 \\
\hline 5. Si hubiera menos demanda o presión asistencial & $3,651(0,718)$ & 0,361 \\
\hline 6. Si no tuviera que hacer asistencia domiciliaria & $2,834(1,115)$ & 0,334 \\
\hline 17. Si tuviera más vacaciones & $3,910(0,890)$ & 0,445 \\
\hline Presión del trabajo (Dimensión 6) & $3,728(0,833)$ & \\
\hline 10. Si pudiera NO hacer partes de baja-alta por enfermedad & $3,40(0,86)$ & 0,368 \\
\hline $\begin{array}{l}\text { 11. Si pudiera NO hacer otros trámites administrativos-burocráticos (partes de in- } \\
\text { capacidad...) }\end{array}$ & $3,66(0,88)$ & 0,370 \\
\hline $\begin{array}{l}\text { 42. Si cada nivel de atención (primaria-especializada) gestionara los procedimien- } \\
\text { tos administrativos de su nivel }\end{array}$ & $4,17(0,78)$ & 0,560 \\
\hline Relaciones con los pacientes (Dimensión 7) & $4,087(0,728)$ & \\
\hline 18. Si los pacientes se preocuparan más de su propia salud & $4,14(0,78)$ & 0,522 \\
\hline 19. Si los pacientes siguieran las recomendaciones/tratamientos que aconsejo & $4,16(0,71)$ & 0,571 \\
\hline $\begin{array}{l}\text { 46. Si pudiera prescindir de realizar algunas actividades (Ej. consejo médico) que } \\
\text { por mi propia experiencia el paciente no escucha ni hace esfuerzos al respecto }\end{array}$ & $3,20(1,01)$ & 0,311 \\
\hline Relaciones con los jefes (Dimensión 8) & $3,946(0,887)$ & \\
\hline 14. Si mis jefes valoraran el trabajo y el esfuerzo & $4,16(0,81)$ & 0,577 \\
\hline 15. Si mis jefes supieran que existo (por ej. mediante una llamada telefónica) & $3,73(0,96)$ & 0,460 \\
\hline Satisfacción con condiciones de trabajo (Dimensión 9) & $3,35(0,83)$ & \\
\hline 20. Si la consulta fuera más espaciosa-luminosa & $3,35(0,83)$ & 0,378 \\
\hline
\end{tabular}

Los ítems 35 y 45 presentan las medias más altas, por encima de 4,20.

Las medias de puntuación entre individuos con mayor y menor rango de satisfacción (percentil 25 y 75) fueron estadística- mente diferentes en todos los ítems, lo cual confirma su capacidad discriminatoria. Todos los coeficientes de correlación fueron significativamente distintos de 0 y de signo negativo (Tabla 2). 
Tabla 2. Análisis de la potencia discriminante de los ítems. Capacidad de cada ítem para diferenciar el grupo de mayor puntuación del de menor puntuación

\begin{tabular}{|c|c|c|}
\hline Ítem & U de Mann-Whitney & $\mathbf{z}$ \\
\hline 1 & 2270,5 & $-7,357$ \\
\hline 2 & 2509,0 & $-6,825$ \\
\hline 3 & 2570,5 & $-6,642$ \\
\hline 4 & 2567,0 & $-6,706$ \\
\hline 5 & 2768,5 & $-7,138$ \\
\hline 6 & 2450,5 & $-6,817$ \\
\hline 7 & 4059,0 & $-4,354$ \\
\hline 8 & 3147,5 & $-5,235$ \\
\hline 9 & 2482,5 & $-7,028$ \\
\hline 10 & 3108,0 & $-5,23$ \\
\hline 11 & 2876,0 & $-5,800$ \\
\hline 12 & 2025,5 & $-8,072$ \\
\hline 13 & 1157,0 & $-10,146$ \\
\hline 14 & 1224,5 & $-10,09$ \\
\hline 15 & 2199,5 & $-7,522$ \\
\hline 16 & 1071,5 & $-10,256$ \\
\hline 17 & 1996,5 & $-8,033$ \\
\hline 18 & 1502,5 & $-9,396$ \\
\hline 19 & 1388,0 & $-9,737$ \\
\hline 20 & 2704,5 & $-6,457$ \\
\hline 21 & 2263,0 & $-7,398$ \\
\hline 22 & 2174,5 & $-7,612$ \\
\hline 23 & 2217,5 & $-7,465$ \\
\hline 24 & 1666,0 & $-8,805$ \\
\hline
\end{tabular}

\begin{tabular}{|c|c|c|}
\hline Ítem & U de Mann-Whitney & $\mathbf{z}$ \\
\hline 25 & 2083,0 & $-7,891$ \\
\hline 26 & 2357,0 & $-7,144$ \\
\hline 27 & 2057,5 & $-7,923$ \\
\hline 28 & 1933,5 & $-8,170$ \\
\hline 29 & 1911,0 & $-8,233$ \\
\hline 30 & 1160,5 & $-10,118$ \\
\hline 31 & 1054,0 & $-10,27$ \\
\hline 32 & 1675,5 & $-8,835$ \\
\hline 33 & 1064,5 & $-10,330$ \\
\hline 34 & 2701,5 & $-6,264$ \\
\hline 35 & 2231,0 & $-7,689$ \\
\hline 36 & 1613,5 & $-9,217$ \\
\hline 37 & 1893,0 & $-8,356$ \\
\hline 38 & 1657,5 & $-8,895$ \\
\hline 39 & 2216,0 & $-7,547$ \\
\hline 40 & 1922,0 & $-8,236$ \\
\hline 41 & 1294,5 & $-9,798$ \\
\hline 42 & 2010,0 & $-8,069$ \\
\hline 43 & 2270,0 & $-7,566$ \\
\hline 44 & 2639,5 & $-6,555$ \\
\hline 45 & 1423,0 & $-9,630$ \\
\hline 46 & 2590,0 & $-6,529$ \\
\hline 47 & 1184,0 & $-10,211$ \\
\hline
\end{tabular}

El test de adecuación de la muestra de Kaiser-Meyer-Olkin fue de 0,903, y el test de esfericidad de Bartlett fue significativo $(p<0,001)$, de modo que se daban las condiciones adecuadas para realizar el análisis factorial.

El total de ítems se agrupó tras el análisis factorial en 9 dimensiones (Tabla 1) oscilando entre 1 y 16 el número de ítems por dimensión. Las dimensiones "Relaciones con los pacientes" y "Satisfacción con la gestión del trabajo" obtuvieron los valores

medios más altos. En cada dimensión se incluyeron los ítems con mayor saturación y se interpretó el contenido de los mismos para configurar cada una de las dimensiones consideradas.

El análisis factorial identificó 5 dimensiones que cumplían con los requisitos de tener un autovalor igual o mayor que $1 \mathrm{y}$ que además aportaran, al menos, un $4 \%$ del total de la varianza. Estos 5 valores de manera conjunta explicaban el 41,287 \% de la varianza total (Tabla 3). 
Tabla 3. Validez de constructo. Resultados del análisis factorial

\begin{tabular}{|c|c|c|c|}
\hline Dimensión & Denominación & $\%$ de la Varianza & Ítems \\
\hline 1 & Competencia profesional & 14,510 & $\begin{array}{c}7(0,588)-8(0,564)-12(0,532)-21(0,500)- \\
23(0,420)-25(0,596)-26(0,433)-27(0,614)- \\
34(0,392)-35(0,531)-36(0,576)-38(0,433)- \\
41(0,469)-43(0,614)-44(0,566)-47(0,562)\end{array}$ \\
\hline 2 & $\begin{array}{l}\text { Satisfacción intrínseca por } \\
\text { la actividad laboral }\end{array}$ & 13,720 & $\begin{array}{c}9(0,621)-13(0,486)-16(0,577)-22(0,446)- \\
24(0,585)-28(0,620)-29(0,530)-30(0,665)- \\
31(0,655)-32(0,559)-33(0,701)-37(0,411)- \\
45(0,500)\end{array}$ \\
\hline 3 & $\begin{array}{l}\text { Relaciones interpersonales } \\
\text { con los compañeros }\end{array}$ & 4,593 & $3(0,822)-4(0,805)$ \\
\hline 4 & $\begin{array}{l}\text { Satisfacción con la gestión } \\
\text { del trabajo }\end{array}$ & 4,420 & $39(0,789)-40(0,779)$ \\
\hline 5 & $\begin{array}{l}\text { Retribución y promoción } \\
\text { profesional }\end{array}$ & 4,044 & $1(0,682)-2(0,541)-5(0,361)-6(0,449)-17(0,428)$ \\
\hline
\end{tabular}

La consistencia de la escala se evaluó mediante el coeficiente alfa de Cronbach obteniendo un valor global de 0,933 . Este coeficiente osciló en las diferentes profesiones entre 0,920 y 0,947 , comprobándose que posee una elevada coherencia interna (Tabla 4). El cálculo de fiabilidad de las dos mitades se realizó correlacionando la suma de los ítems pares con la suma de los impares. El coeficiente total obtenido fue de 0,882 .

Tabla 4. Índices de fiabilidad por perfil profesional

\begin{tabular}{lccccc}
\hline Profesión & No sujetos & Media (+) & DT & $\begin{array}{c}\text { Índice de fiabilidad } \\
\text { alfa de Cronbach }\end{array}$ & $\begin{array}{c}\text { Índice de fiabilidad } \\
\text { de dos mitades }\end{array}$ \\
\hline Médicos & 188 & 180,01 & 18,04 & 0,920 & 0,850 \\
\hline Pediatras & 48 & 183,24 & 18,09 & 0.926 & 0,855 \\
\hline Enfermeras & 174 & 177,07 & 21,32 & 0,947 & 0,915 \\
\hline Perdidos & 4 & & & & $\mathbf{0 , 9 8 2}$ \\
\hline Total & $\mathbf{4 1 4}$ & $\mathbf{1 7 9 , 0 9}$ & $\mathbf{1 9 , 5 2}$ & $\mathbf{0 3 3}$ & \\
\hline
\end{tabular}

(+) Puntuación media del cuestionario

DT: Desviación típica de la puntuación media por perfil profesional

Los coeficientes alfa de Cronbach de las dimensiones (Tabla 5) se situaron en- tre 0,569 (Dimensión 4) y 0,911 (Dimensión 3).

Tabla 5. Características de las Dimensiones del cuestionario y homogeneidad o consistencia de las mismas

\begin{tabular}{|c|c|c|}
\hline Dimensión & Mediana & alfa-Cronbach \\
\hline Competencia profesional (Dimensión 1) & 4 & 0,864 \\
\hline Satisfacción intrínseca por la actividad laboral (Dimensión 2) & 3 & 0,872 \\
\hline Relaciones interpersonales con los compañeros (Dimensión 3) & 4 & 0,911 \\
\hline Satisfacción con la gestión del trabajo (Dimensión 4) & 4 & 0,569 \\
\hline Retribución y promoción profesional (Dimensión 5) & 3 & 0,619 \\
\hline
\end{tabular}




\section{DISCUSIÓN}

Esta es la primera vez que en España se ha desarrollado un instrumento de mejora de la satisfacción laboral, lo cual genera el inconveniente de que no permite realizar comparaciones.

Aunque siempre es deseable una elevada tasa de respuesta, en estudios realizados mediante cuestionarios enviados por correo es difícil superar el $50 \%$. En nuestro caso, el índice de respuesta obtenido es aceptable $(62,3 \%)$ y es equivalente al logrado en encuestas realizadas en médicos generales ${ }^{35-36}$ e incluso superior al resultado obtenido en el medio hospitalario ${ }^{37}$.

Hubiese sido de interés considerar también la opinión de los profesionales no sanitarios, pero el contenido del cuestionario no es apropiado en su totalidad para obtener dicha información y además hubiera sido necesario ampliarlo, lo que hubiera complicado su cumplimentación ${ }^{37}$.

Algunos factores pueden mediar e influir en la tasa de respuesta del cuestionario como son la recomendación de cumplimentarlos tras su presentación en una sesión de equipo, problemas interpersonales entre los miembros del equipo y el hecho de que la fecha de su realización pueda haber limitado una mayor cobertura al coincidir con fechas consideradas vacacionales.

La validez de contenido señala el grado en que la medición abarca todas o la mayoría de las dimensiones del concepto que se quiere estudiar y su valoración es subjetiva. Consideramos adecuada la validez de contenido por haberse basado en revisión bibliográfica sobre el tema y en la opinión de profesionales con experiencia en investigación en atención primaria.

Los resultados muestran que el cuestionario desarrollado en este estudio es un instrumento con suficiente validez y fiabilidad para medir aquello para lo que el cuestionario está desarrollado, presentando una buena consistencia interna.

La validez de constructo determina el grado en que la medición se corresponde con los conceptos que quiere medir. El análisis factorial confirmó la existencia de 9 dimensiones, que se corresponden mayoritariamente con las propuestas en el diseño del cuestionario, demostrando su validez de constructo. La Dimensión denominada "Satisfacción con la gestión del trabajo" es la más importante de la escala, ya que explica el $22,4 \%$ de la variabilidad.

El análisis factorial de los componentes principales confirmó la validez de contenido del cuestionario. Indica que cada conjunto de preguntas de cada Dimensión midió internamente un mismo concepto, lo que constituye un requisito esencial para otorgar validez al cuestionario ${ }^{38}$.

Algunos autores señalan que una solución factorial es satisfactoria si explica por lo menos el 50 \% de la varianza total y generalmente se espera que la primera Dimensión reúna los aspectos mas relevantes del constructo y acumule el mayor porcentaje de la varianza ${ }^{39}$.

En conjunto, las 5 dimensiones explican más del $40 \%$ de la variabilidad total del instrumento, nivel que suele exigirse como criterio de validez estructural $^{40}$.

En cuanto a la fiabilidad, el índice que señala mayor consistencia interna u homogeneidad es el alfa de Cronbach. La consistencia interna de una escala se considera aceptable cuando se encuentra entre 0,7 y $0,9^{41-42}$. Valores de consistencia interna inferiores a 0,7 indican una pobre correlación entre los ítems y aquellos por encima de 0,9 se observan cuando hay más de 20 ítems ${ }^{43}$, que sobreestiman el coeficiente, recomendando el uso de diferentes subescalas $^{44} \mathrm{o}$ bien reduciendo el número de ítems. También, cuando la consistencia está por encima de 0,9 puede ser indicador de redundancia o duplicación de ítems que midan el mismo aspecto del constructo ${ }^{45}$ que en esta escala alcanza 0,92 , llegando a 0,928 para el grupo de médicos. El índice de consistencia interna obtenido en nuestro trabajo muestra la consistencia interna del cuestionario utilizado.

El número de dimensiones es directamente proporcional al número de ítems y se espera que una dimensión esté formada por un número mínimo de ítems ${ }^{39}$.

Tanto la consistencia interna como la solución factorial de una escala, varían de 
una población a otra ${ }^{39,42,43}$. Por tanto, siempre es necesario corroborar el comportamiento psicométrico de una escala en una población particular antes de su uso en diferentes contextos de la atención y el cuidado de los servicios de salud.

El proceso de validación es un proceso continuo en constante evaluación y siempre es necesario hacer adaptaciones cuando el contexto cultural es distinto ${ }^{46-47}$.

El cuestionario presenta unos buenos índices de discriminación en la mayoría de los ítems.

Entre las limitaciones del estudio se destaca el de la representatividad de la muestra, debido a que estuvo limitada a aquellos a los que se distribuyó el cuestionario lo cual a su vez depende del director del equipo y la fecha de realización en verano.

El análisis factorial halló 5 dimensiones capaces de explicar el $41,6 \%$ de la varian$\mathrm{za}$, por lo cual podemos considerar que las puntuaciones tienden a agruparse en torno a factores reales de un determinado constructo, lo que apoya la validez de la escala en este sentido.

En la gran cantidad de publicaciones sobre satisfacción laboral de los últimos años $^{48-49}$, se observa una tendencia a tratar de diseñar cuestionarios cada vez más específicos, cortos y vinculados a aquellos aspectos concretos de la satisfacción que se quiere estudiar. Los cuestionarios generales al estilo Font-Roja están quedando desfasados, lo cual es coherente con la última edición de los estándar ${ }^{48-50}$.

El cuestionario que se propone, suple la carencia existente y permite ofrecer una herramienta para evaluar el comportamiento del profesional.

Como propuestas de futuro, convendría validar el cuestionario en otras poblaciones más amplias para valorar mejor su utilidad.

Contar con un instrumento validado localmente es fundamental para analizar el grado de satisfacción laboral y comparar su evolución después de la eventual implementación de medidas para mejorar su relación laboral.

\section{BIBLIOGRAFÍA}

1. Sibbald B, Enzer I, Cooper C, Routh U, Sutherland V. GP job satisfaction in 1987, 1990 and 1998: lessons for the future? Fam Pract 2000; 17: 364-71.

2. Newmank, Maylor U. The NHS Plan: nurse satisfaction, commitment and retention strategies. Health Serv Mange Res 2002; 15: 93-105.

3. FiRTh-Cozens J. Interventions to improve physicians' well-being and patient-care. Soc Sci Med 2001; 52: 215-222.

4. Villares J, Ruiz A, López M, SÁinz R. La satisfacción profesional en el equipo de atención primaria: oportunidades de mejora. Cuadernos de Gestión 2000; 6: 60-67.

5. Bernat A, IzQuierdo P, Jiménez L, Bardón I, Casado N. Satisfacción laboral: Análisis de las variables predictoras en una muestra de profesionales de la salud, en Atención Especializada, de un área sanitaria de la Comunidad de Madrid. Med Segur Trab (Internet) 2009; 55: 4956.

6. Villagómez A, Hernández C, Villareal R. Satisfacción laboral en médicos a seis años de distancia. Rev Med IMSS 2003; 41: 399-405.

7. MECHANIC D. Physician discontent: challenges and opportunities. JAMA 2003; 290: 941-946.

8. VALENCIA M. Propuesta para medir la relación supervisión-satisfacción laboral en enfermería. Rev Enferm IMSS 2001; 9: 81-84.

9. Horowitz C, Suchman A, Branch W, Frankel R. What do doctors find meaningful about their work? Ann Intern Med 2003; 138: 772-775.

10. Devoe J, Fryer G, Lee H, Phillips R, Green L. Does career dissatisfaction affect the ability of family phisicians to deliver high-quality patient care? J Fam Pract 2002; 51: 223-228.

11. Sмiтн R. Why are doctors so unhappy? There are probably many causes, some of them deep. Br Med J 2001; 322: 1073-1074.

12. Rodríguez Fernández-Oliva CR, SuÁrez López de VERGara RG. Problemas profesionales. Percepción de los pediatras de atención primaria de Tenerife. BSCP Can Ped 2005: 29, 2: 151-154.

13. PІко B. Burnout, role conflict, job satisfaction and psychosocial health among Hungarian health care staff: a questionnaire survey. Int J Nurs Stud 2006; 43: 311-318.

14. Lomeña J, Campaña F, Nuevo G, Rosas D, Berrocal A, GARCíA F. Burnout y satisfacción laboral en atención primaria. Med Fam Andal 2004; 5: 147-155.

15. Simón M, Blesa A, Bermejo C, Calvo M, Gómez C. Estresores laborales y satisfacción en la enfermería de una unidad de críticos. Enferm Intensiva 2005; 16: 3-14. 
16. Freeborn D, Hooker R, Pope c. Satisfaction and well-being of primary care providers in managed care. Eval Health Prof 2002; 25: 239-254.

17. Comité director del Plan Estratégico del Área 11 de Atención Primaria de Madrid. El Plan Estratégico en un área de atención primaria: una propuesta metodológica. Rev Adm Sanit 2001; 5: $71-83$.

18. Hernández R, Fernández-Collado C, Baptista P. Metodología de la investigación. Cuarta edición. México: McGraw-Hill Interamericana; 2006.

19. Lamprea J, Gómez-Restrepo C. Validez en la evaluación escalas. Rev Colomb Psiquiatr 2007; 36: 340-348.

20. Kaplan R, Saccuzzo D. Pruebas psicológicas. Sexta edición. México: Thomson; 2006.

21. Cortina J. What is coefficient alpha? An examination of theory and applications. J Appl Psychol 1993; 78: 98-104.

22. Gliner J, Morgan G, Harmon R. Measurement reliability. J Am Acad Child Adolesc Psychiatry 2001; 40: 486-488.

23. Peiró J, Silla I, Sanz T, Rodríguez J, García J. Satisfacción laboral de los profesionales de atención primaria. Psiquis 2004; 25: 129-140.

24. Aranaz J, Mira J. El cuestionario Font Roja. Un instrumento de medida de la satisfacción en el medio hospitalario. Todo Hosp 1988; 52: 63-66.

25. Peiró J, González V, Zurriaga R, Ramos J, Bravo M. El cuestionario de Satisfacción Laboral de Profesionales de la Salud de Equipos de Atención Primaria (CSLPS-EAP). Rev Psicol Salud 1989; 1: 135-174.

26. Martín J, Cortés J, Morente M, Caboblanco M, GaRIJO J, Rodríguez A. Características métricas del Cuestionario de Calidad de Vida Profesional (CVP-35). Gac Sanit 2004; 18: 129-136.

27. Robles M, Dierssen T, Martínez E, Herrera P, Díaz A, LloRCA J. Variables relacionadas con la satisfacción laboral: un estudio transversal a partir del modelo EFQM. Gac Sanit 2005; 19: 127-134.

28. MASLACH C. Understanding burnout. En: Payne S, editor. Job stress and burnout. Deary I, Agius R, Sadler A. Personality and stress in consultant psychiatrist. Londres: Sage; 1982: p. 20.

29. Melí J, Pradilla J, Martí N, Sancerni M, Oliver A, Tomás J. Estructura factorial, fiabilidad y validez del cuestionario de satisfacción S21/26: un instrumento con formato dicotómico orientado al trabajo profesional. Rev Psicol Univ Tarracon 1990; 12: 25-39.

30. Melí J, Peiró J. El cuestionario de satisfacción S10/12:estructura factorial, fiabilidad y validez. Rev Psicol Trab Org 1989; 4: 179-187.

31. CABEZAS C. La calidad de vida de los profesionales. FMC 2000; 7: 53-68.

32. Stevens J. Power of the multivariate analysis of variance tests. Psychol Bull 1980; 88: 728-737.
33. KAISER H. An index of factorial simplicity. Psychometrica 1974; 34: 31-36.

34. BARTLETT M. Test of significance in factor analysis. Br J Psychol 1950; 3: 77-85.

35. Cortés J, Martín J, Morente M, Caboblanco M, Garijo J, Rodríguez A. Clima laboral en atención primaria. ¿Qué hay que mejorar? Aten Primaria 2003; 32: 288-295.

36. Mira J, Vitaller J, Buil J, Aranaz J, Rodríguez-Marín J. Satisfacción y estrés laboral en médicos generalistas del sistema público de salud. Aten Primaria 1994; 14: 1135-1140.

37. Mozota JR, Ansorena L, Rebollo H, Antolín I. Resumen de la Encuesta de Satisfacción del Personal en el Hospital Nacional Valdecilla. Todo Hosp 1990; 67:21-28

38. Batista-Foguet J, Coenders G, Alonso J. Análisis factorial confirmatorio. Su utilidad en la validación de cuestionarios relacionados con la salud. Med Clin (Barc) 2004; 122 (Supl 1): 21-27.

39. Gorsuch R. Exploratory factor analysis: its role in item analysis. J Pers Asses 1997; 68: 532-560.

40. Moret L, Chwalow J, Baudoin-Balleur C. Evaluer la qualité construction dùne échelle. Rev Epidem Santé Publ 1993; 41: 65-71.

41. Cervantes V. Interpretaciones del coeficiente de alpha de Cronbach. Avances en Medición 2005; 3: 9-25.

42. Velarde E, Ávila C. Consideraciones metodológicas para evaluar la calidad de vida. Salud Pública Mex 2002; 44: 448-463.

43. Campo-Arias A, Oviedo H. Propiedades psicométricas de una escala: la consistencia interna. Rev Salud Pública 2008; 10: 831-839.

44. CAMPO-Arias A. Usos del coeficiente de alfa de Cronbach (carta al editor). Biomedica 2006; 26: 585-588.

45. JAJU A, CRASK M. The perfect design: optimization between reliability, validity, redundancy in scale items and response rates. Am Market Assoc 1999; 10: 127-131.

46. Carvajal A, Centeno C, Watson R, Martínez M, SAnz Rubiales A. Cómo validar un instrumento de medida de la salud. An Sist Sanit Navar 2011; 34: 63-72.

47. Campo-Arias A, Oviedo H. Propiedades psicométricas de una escala: la consistencia interna. Rev Salud Pública 2008; 10: 831-839.

48. Herencia A, Prieto A, March J. Las falacias presentes en la medición de la satisfacción laboral. Rev Calidad Asistencial 2001; 16: 61-65.

49. Thompson B. Educational and psychological measurement 2000. Disponible en: http://www. sagepub.co.uk/journals/details/j0038.html

50. The Society for Industrial and Organizational Psychology (US). Standards for Educational and Psychological Testing. Berkeley: APA, 1999. 


\section{ANEXO 1}

Escala de evaluación de mejora de la satisfacción laboral (CMSL) en profesionales de atención primaria*

Mi satisfacción laboral mejoraría... [Puntuación posible: 1, 2, 3, 4, 5]

1. Totalmente en desacuerdo; 2. En desacuerdo; 3 . Indiferente; 4. De acuerdo; 5 . Totalmente de acuerdo $\begin{array}{lllll}1 & 2 & 3 & 4 & 5\end{array}$

1. Si mi sueldo fuera superior

2. Si hubiera posibilidades de promoción

3. Si mi relación con los compañeros médicos/enfermeras del EAP fuera mejor

4. Si mi relación con otros compañeros no médicos/enfermeros del EAP fuera mejor

5. Si hubiera menos demanda o presión asistencial

6. Si no tuviera que hacer asistencia domiciliaria

7. Si dedicara un tiempo mínimo, en horario laboral, para mi formación

8. Si pudiera actuar en actividades de investigación

9. Si pudiera eliminar "de mi cupo" unos pocos pacientes con quienes no logro empatizar

10. Si pudiera NO hacer partes de baja-alta por enfermedad

11. Si pudiera NO hacer otros trámites administrativos-burocráticos (partes de incapacidad, certificado de reconocimiento)

12. Si mis relaciones con atención especializada fueran mas ágiles y fluidas

13. Si mis pacientes valoraran el trabajo y el esfuerzo

14. Si mis jefes valoraran el trabajo y el esfuerzo

15. Si mis jefes supieran que existo (por ej. mediante una llamada telefónica)

16. Si fuera capaz de trabajar sin hacer una medicina defensiva

17. Si tuviera más vacaciones

18. Si los pacientes se preocuparan más de su propia salud

19. Si los pacientes siguieran las recomendaciones/tratamientos que aconsejo

20. Si la consulta fuera más espaciosa-luminosa

21. Si tuviera facilidad de mis jefes para acudir a congresos y actividades de formación

22. Si sintiera un menor control (gasto farmacia) de mis superiores

23. Si pudiera trabajar más con programas y menos con demanda de atención aguda

24. Si los pacientes no "me presionaran" para realizar actividades que considero injustificadas (bajas injustificadas, Rx y analítica injustificadas...)

25. Si pudiera disponer de más tiempo para cada paciente

26. Si las cargas de trabajo entre los miembros del equipo (médico-enfermera) fueran más equitativas, con una correcta distribución de las tareas a realizar por los diferentes estamentos

27. Si trabajáramos en equipo

28. Si mi trabajo no fuera tan monótono (mismos pacientes y mismos problemas)

29. Si tuviera flexibilidad para las agendas de citación

30. Si pudiera eliminar los hiperfrecuentadores

31. Si pudiera trabajar en un ambiente tranquilo, sin amenazas ni coacciones

32. Si pudiera desconectar al acabar la jornada

33. Si mis pacientes fueran, realmente, con problemas de salud

34. Si tuviera premios y/o reconocimiento a la labor bien desarrollada

35. Si hubiera coordinación entre servicios sanitarios y servicios sociales

36. Si pudiera trabajar sin estrés, sin prisas ni agobios

37. Si pudiera conciliar vida laboral y familiar

38. Si pudiera ser mas ágil en aportar solución a mis pacientes

39. Si pudiera agilizar las listas de espera para pruebas diagnósticas

40. Si pudiera agilizar las consultas con especialistas

41. Si mis propuestas fueran escuchadas y estudiadas-aplicadas por mis superiores

42. Si cada nivel de atención (primaria-especializada) gestionara los procedimientos administrativos de su actividad (baja laboral, pruebas diagnósticas.)

43. Si hubiera planes de formación en farmacoterapia

44. Si hubiera modelos de seguimiento de los pacientes polimedicados

45. Si pudiera eliminar la demanda injustificada

46. Si pudiera prescindir de realizar algunas actividades (ej: consejo médico) que, por mi propia experiencia, el paciente no me escucha y no hace el más mínimo esfuerzo

47. Si pudiera contactar con especialistas que puedan resolver dudas "en el momento"

${ }^{*}$ El intervalo de puntuaciones puede ir de 47 a 235 puntos. 